\title{
Oxygen Abundances: New Results from [O I] Lines
}

\author{
Christopher Sneden
}

Department of Astronomy, University of Texas, Austin, TX 78712, USA

\author{
Francesca Primas \\ European Southern Observatory, Karl-Schwarzschild-Strasse 2, D-85748 \\ Garching bei München, Germany
}

\begin{abstract}
From new high resolution spectra of metal-poor giants we use equivalent widths of [O I], Sc II, and Fe II lines to derive new values of $[\mathrm{O} / \mathrm{Sc}]$ and $[\mathrm{O} / \mathrm{Fe}]$. These results are generally consistent with previous determinations of oxygen abundances from $[\mathrm{O} \mathrm{I}]$ lines, but the star-to-star scatter is greatly reduced.
\end{abstract}

\section{Introduction}

Conti et al. (1967) first suggested that $[\mathrm{O} / \mathrm{Fe}]$ ratios in metal-poor stars are substantially larger than the Solar System value. To reach this conclusion they analyzed the [O I] $6300,6363 \AA$ lines in a sample of bright giants of mainly the young and old disk. This work led Lambert, Sneden, \& Ries (1974) to search for the $6300 \AA$ line in the very metal-poor $([\mathrm{Fe} / \mathrm{H}] \sim-2.7)$ giant $\mathrm{HD} 122563$. Their successful detection of the line implied a very high abundance, $[\mathrm{O} / \mathrm{Fe}] \simeq+0.6$. Subsequently, Sneden, Lambert, \& Whitaker (1979) found large $[\mathrm{O} / \mathrm{Fe}]$ ratios from analyses of the O I very high excitation $(9.15 \mathrm{eV}) 7770 \AA$ triplet lines in a number of metal-poor dwarfs. Oxygen abundance studies have continued to the present, and there is unanimous agreement that $[\mathrm{O} / \mathrm{Fe}]>0$ in almost all low metallicity stars. But abundances deduced via LTE analyses of different oxygenspecies transitions do not agree in detail. The $[\mathrm{O} \mathrm{I}]$ lines suggest $[\mathrm{O} / \mathrm{Fe}] \simeq+0.4$, essentially independent of metallicity in the range $-1.0 \geq[\mathrm{Fe} / \mathrm{H}] \geq-2.7$ (e.g., Gratton et al. 2000 and references therein), and new data for the $\mathrm{OH}$ infrared vibration-rotation bands (Balachandran \& Carney 1996, Melendez, Barbuy, \& Spite 2000) are consistent with that result. But the $\mathrm{O} I$ triplet lines and $\mathrm{OH}$ nearUV electronic band lines yield increasing oxygen abundances with decreasing metallicity, approaching $[\mathrm{O} / \mathrm{Fe}] \sim+1$ at $[\mathrm{Fe} / \mathrm{H}] \sim-3$ (Israelian, García-Lopez, \& Rebolo 1998; Boesgaard et al. 1999).

Here we make no attempt to solve the oxygen abundance problem in low metallicity stars. Instead, we re-examine the abundances deduced from the [O I] lines in giant stars, using an analytical technique employed by Lambert et al. (1974) and subsequently by, e.g., Gratton \& Ortolani (1986), but relatively neglected in recent years: a comparison of the relative strengths of $[\mathrm{O} I]$ and Sc II lines. 


\section{Observations, Reductions, Analyses}

The spectra originally were obtained for other projects with the McDonald Observatory $2.7 \mathrm{~m}$ Smith reflector and the Tull "2d-coudé" echelle spectrometer. The spectra have resolving power $R \simeq 60,000$, and $S / N>100$. In the reduction procedures, care was taken in the cancellation (via division by the spectrum of a rapidly rotating hot star) of the telluric $\mathrm{O}_{2}$ features surrounding the stellar [O I] $6300 \AA$ line. Radial velocity shifts moved the stellar [O I] lines away from the night sky emission lines in all cases. In the top panel of Figure 1 we show examples of program star spectra near the $6300 \AA$ line.

Neighboring lines are also labeled, including the Sc II $6300.67 \AA$ feature. As has been noted in earlier papers, the [O I] and Sc II lines have very similar equivalent widths (EWs) in the Sun and solar metallicity giant stars, so the apparent larger strength of the $[\mathrm{O} \mathrm{I}]$ line here points to an oxygen overabundance.

We measured the EWs of the [O I] $6300 \AA$ line (and the $6363 \AA$ line when it was strong enough), as many as seven Sc II lines, and as many as six Fe II lines in the yellow-red spectral region of our spectra. Only these transitions were considered, first because several investigations (notably that of Thévenin \& Idiart 1999) suggest that neutral species of metals such as Fe I (but not the ionized species) may be subject to substantial departures from LTE in the atmospheres of metal-poor stars. Second, O I has a high ionization potential, and therefore is the majority species of oxygen, as are the ionized species of scandium and iron. The [O I] and Sc II line excitation potentials differ by only $\simeq 1.5 \mathrm{eV}$, and so the dependence on atmospheric uncertainties in ratios of abundances derived from these transitions is very small (e.g. Lambert et al. 1974).

The measured EWs were employed in a standard LTE abundance analysis (e.g., Westin et al. 1999), the results of which are summarized in the lower panel of Figure 1. A dashed straight line has been drawn by hand to represent the mean trend of the abundances. The residual scatter about this line is small, with $\sigma \simeq 0.1$. A similar plot could be shown of the $[\mathrm{O} / \mathrm{Fe}]$ trend with $[\mathrm{Fe} / \mathrm{H}]$, but the results would look quite similar to that shown for $[\mathrm{Sc} / \mathrm{Fe}]$, since our derived $[\mathrm{Sc} / \mathrm{Fe}]$ do not differ significantly from zero.

The abundances are very consistent with previous [O I] studies in the metallicity regime $[\mathrm{Fe} / \mathrm{H}]>-2$, and shows evidence for a small upward trend at lower metallicities. But the oxygen abundances even at the lowest $[\mathrm{Fe} / \mathrm{H}]$ values of our sample remain incompatible with the much larger abundances derived from the $\mathrm{O}$ I triplet and UV OH transitions.

Acknowledgments. We gratefully acknowledge research support through NSF grant AST-9987162.

\section{References}

Balachandran, S., \& Carney, B. W. 1996, AJ, 111, 946

Boesgaard, A. M., King, J. R., Deliyannis, C. P., \& Vogt, S. S. 1999, AJ, 117, 492 

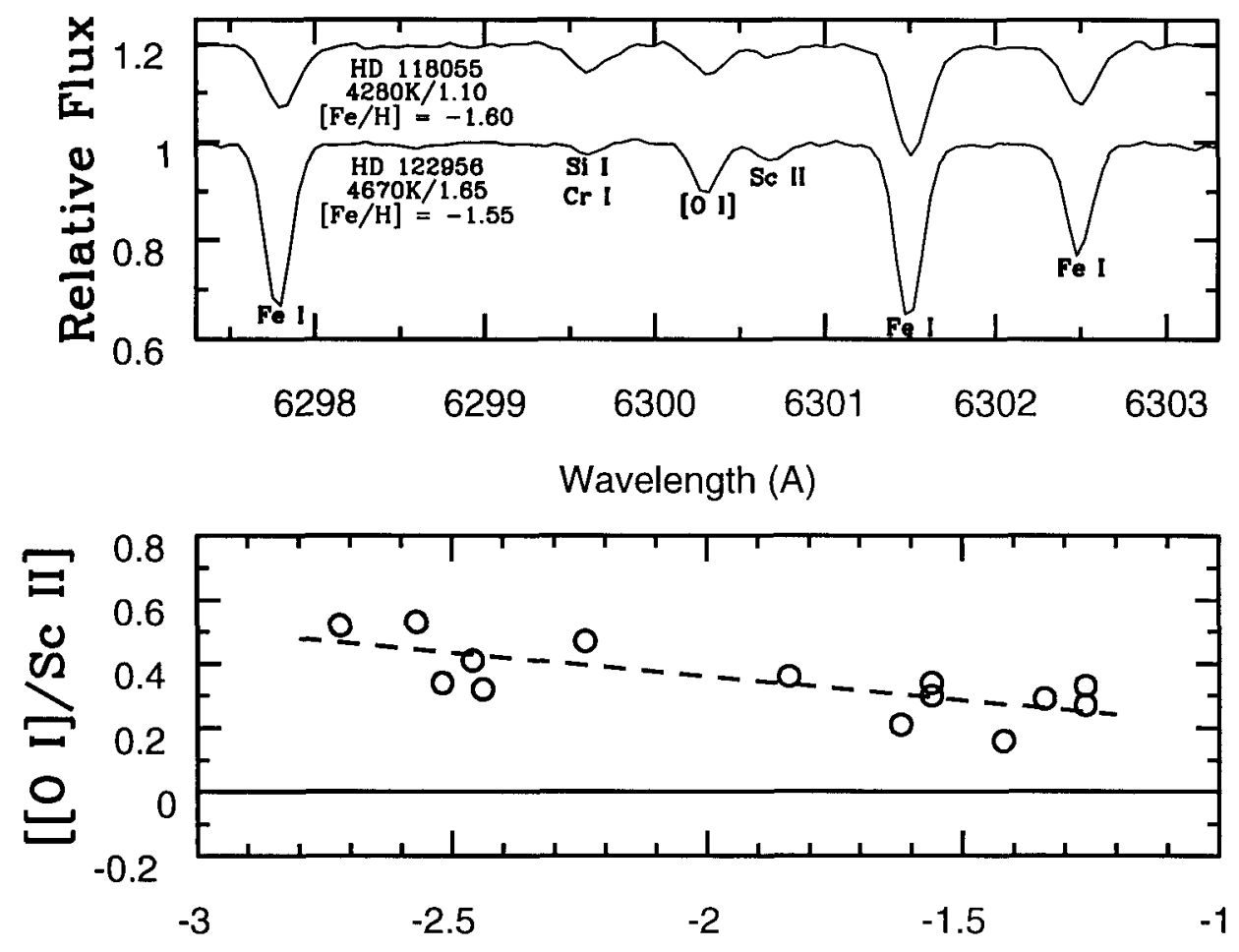

$[\mathrm{Fe} \mathrm{II} / \mathrm{H}]$

Figure 1. Upper panel: sample spectra of two of the program stars. The relative flux is correct for HD 122956, and the spectrum of HD 118055 is shifted vertically for display purposes. Bottom panel: $[\mathrm{O} / \mathrm{Sc}]$ values correlated with $[\mathrm{Fe} / \mathrm{H}]$. The axis labels explicitly state the transitions used for these abundances. The dashed line is a handdrawn linear best fit to the data.

Conti, P. S., Greenstein, J. L., Spinrad, H., Wallerstein, G., \& Vardya, M. S. 1967, ApJ, 148, 105

Gratton, R. G., \& Ortolani, S. 1986, A\&A, 169, 201

Gratton, R. G., Sneden, C., Carretta, E., \& Bragaglia, A. 2000, A\&A, 354, 169

Israelian, G., García Lopez, R. J., \& Rebolo, R. 1998, ApJ, 507, 805

Lambert, D. L., Sneden, C., \& Ries, L. M. 1974, ApJ, 188, 97

Melendez, J., Barbuy, B., \& Spite, F. 2000, preprint

Sneden, C., Lambert, D. L., \& Whitaker, R. W. 1979, ApJ, 234, 964

Thévenin, F., \& Idiart, T. P. 1999, ApJ, 521, 753

Westin, J., Sneden, C., Gustafsson, B., \& Cowan, J. J. 2000, ApJ, 530, 783 